\title{
Network Selection in Heterogeneous Wireless Networks based on Fuzzy Multiple Criteria Decision Making
}

\author{
K. Radhika \\ Department of Information Technology \\ CBIT \\ Hyderabad,India
}

\author{
Dr. A. Venugopal Reddy \\ Daprtment of Computer Science \\ Osmania University \\ Hyderabad,India
}

\begin{abstract}
Next generation wireless networks are expected to exhibit heterogeneity in terms of wireless access technologies, services and application requirements. These heterogeneous wireless access networks consist of the integration of various wireless networks especially Wi-Fi (WLAN), Wi-Max and 3G/B3G cellular networks. One of the challenging problems is to choose the optimal network depending upon the type of the demanding application. Vertical handoff occurs when a mobile terminal decides to switch to the selected network from the current network. This paper mainly deals with a network selection algorithm based on Fuzzy Multiple Attribute Decision Making. The algorithm considers the factors of Received Signal Strength(RSS), Monetary $\operatorname{cost}(\mathrm{C})$, Band Width $(\mathrm{BW})$, Velocity $(\mathrm{V})$ and user preference $(\mathrm{P})$. It finds the Network selection function (NSF) that measures the efficiency in utilizing radio resources by handing off to a particular network. The network that provides highest NSF is selected as the best network to hand off from the current access network.
\end{abstract}

\section{General Terms}

Heterogeneous wireless networks, Network Selection, Algorithm

\section{Keywords}

Vertical handoff, MADM, RSS, Cost, Bandwidth, Velocity, User Preference, NSF.

\section{INTRODUCTION}

The development and proliferation of wireless and mobile technologies have revolutionized the world of communications. During the evolution from $2 \mathrm{G}$ to $3 \mathrm{G}$, a range of wireless systems including GPRS, IMT-2000, Bluetooth, WLAN and Hyper LAN have been developed. All these systems were designed independently targeting different service types, data rates and user requirements. These systems have their own merits and shortcomings [1]. The strengths of third generation (3G) cellular networks such as UMTS and CDMA 2000 consist of their global coverage where as their weakness lie in bandwidth capacity and operation costs. On the other hand wireless LAN such as IEEE802.11 offers higher bandwidth with low operation costs, but it covers a relatively short range [2].

Modern wireless communication systems consist of cellular, WLAN and Wi-Max access networks coexisting and are termed heterogeneous wireless networks [3]. Technological advancements in the evolution of portable devices have made possible the support of different radio access technologies. This has raised much interest in the integration and interworking of Wi-Fi (WLAN), WiMax and 3G/B3G cellular networks [2]. Hence it is expected that mobile users could enjoy truly seamless mobility and ubiquitous service access in an always best connected mode, employing the most efficient combination of available access systems at anytime and anywhere. In this context the development of an appropriate interworking solution for these heterogeneous wireless networks is crucial [4].

Wi-Fi or example $802.11 \mathrm{x}$ has the characteristics of high band width, low cost and short distance access; 802.16x or Wi-Max systems are optimized to provide real-time high data rate services in WMAN environments or 3G Cellular WWAN networks are slower, high cost, long range and always connected access. All these systems were designed independently, targeting different service types, data rates, and users. As these systems all have their own merits and shortcomings, there is no single system that is good enough to replace all the other technologies. Instead of putting efforts into developing new radio interfaces and technologies for $4 \mathrm{G}$ systems, which some researchers are doing, we believe establishing $4 \mathrm{G}$ systems that integrate existing and newly developed wireless systems is a more feasible option [1] The integration and inter operation of these heterogeneous networks pose several challenges. One of the important issues is the design of intelligent network selection algorithms that provide better performance to the multi-interface terminals in the integrated networks [5-7].

The IEEE 802.21 standard addresses seamless handover in homogeneous and heterogeneous environments. Information collection and exchange can be performed by the Media Independent Handover (MIH) Services. The IEEE 802.21 standard only provides the capability and possibility of allowing a mobile node to select a suitable network to handover. However, IEEE 802.21 does not have a defined the way of how the networks selection process should be performed [8-11].

This paper proposes a network selection algorithm based on Fuzzy multiple attribute decision making. A handoff algorithm must be capable of making a decision based on incomplete information and in a region of uncertainty. We are designing an adaptive multicriteria handoff decision algorithm that incorporates fuzzy logic because of the inherent strength of fuzzy logic in solving problems exhibiting imprecision and the fact that many of the terms used for describing radio signals are fuzzy in nature [9-11].

Fuzzy logic can be exploited to develop approximate solutions that are both cost-effective and highly useful. In this paper, we assume a scenario where the user is initially connected to a CDMA network and is moving towards a Wi-Max network and finally moves towards a Wi-Fi network.

The algorithm considers the factors of RSS, monetary cost, available band width, mobile terminal velocity and User preference of the 3 candidate networks for choosing the target network. In 
section II, the normalization functions of the above parameters are described. In section III the weight vector corresponding to the specified parameters is computed. The Network Selection Function Value of each network is calculated in section IV. The network with the highest NSF value is chosen as the target network and the mobile terminal hands over to the target network. In section V, we present conclusions and future directions.

\section{FINDING NORMALIZATION FUNCTIONS}

The parameters that are considered for making handover decision are defined as follows:

RSS : The strength of the signal received from the Candidate network.

Monetary Cost (C) : The operating cost of the network.

Band width (BW) : The amount of available band width of the Candidate network.

Velocity (V) : The Velocity that is supported by the Candidate Network.

User Preference $(\mathrm{P})$ : User can select the preference for the Candidate network ranging from 1 to 10 .

The parameters are classified as high parameters and low parameters to facilitate normalization. The value of high parameters should be as high as possible which includes RSS, BW, $\mathrm{V}$ and $\mathrm{P}$. The value of low parameters should be as low as possible that includes $\mathrm{C}$.

The normalization function is given by equation (1)

$N(x)=\frac{x-x_{\min }}{x_{\max }-x_{\min }}$

The normalization function of RSS is given by equation (2)

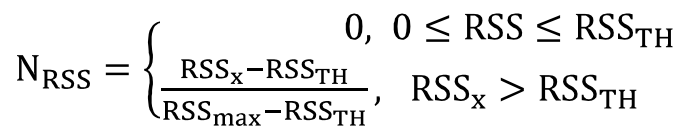

Where $\mathrm{RSS}_{\mathbf{X}}$ is the actual strength of the signal received from the candidate base station. $\mathrm{RSS}_{\mathrm{TH}}$ is defined as the threshold signal strength. $\mathrm{RSS}_{\max }$ is the maximum RSS that can be received from a candidate base station. The normalization function of RSS is plotted in Figure 1.

The normalization function of cost (C) is given by equation (3).

$\mathrm{N}_{\mathrm{C}}=\left\{\begin{array}{c}0, \mathrm{C}_{\mathrm{x}}>\mathrm{C}_{\mathrm{TH}} \\ 1-\frac{\mathrm{C}_{\mathrm{x}}}{\mathrm{C}_{\mathrm{TH}}}, 0 \leq \mathrm{C}_{\mathrm{x}}<\mathrm{C}_{\mathrm{TH}}\end{array}\right.$

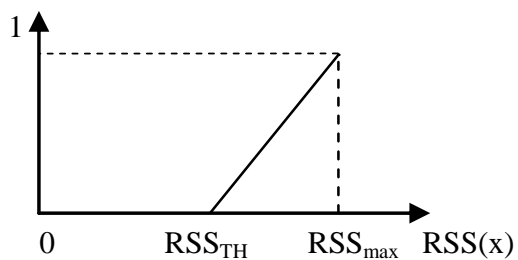

Figure 1. Normalization function of RSS

Where $\mathrm{Cx}$ is the operating cost of the network to which the candidate base station belongs to. $\mathrm{C}_{\mathrm{TH}}$ is the threshold cost, above which it is considered that the network is expensive. The normalization function of Cost is plotted in Figure 2.

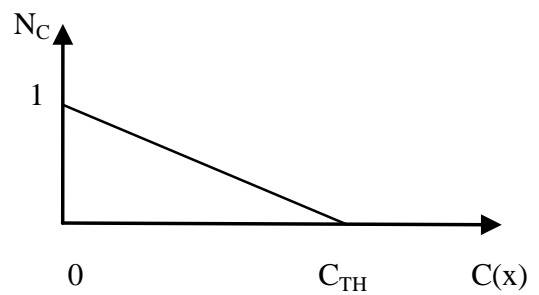

Figure 2. Normalization function of Cost

The normalization function of Bandwidth (BW) is given by equation (4)

$N_{B W}=\left\{\begin{array}{c}0, B_{x}>B_{\max } \\ \frac{B_{x}}{B_{\text {max }}}, 0 \leq B_{x} \leq B_{\max }\end{array}\right.$

Where $B_{X}$ is the required bandwidth of the mobile station and $B_{\text {max }}$ is the maximum bandwidth that can be provided by the base station. The normalization function of the bandwidth is plotted in Figure 3.

$\mathrm{N}_{\mathrm{BW}}$

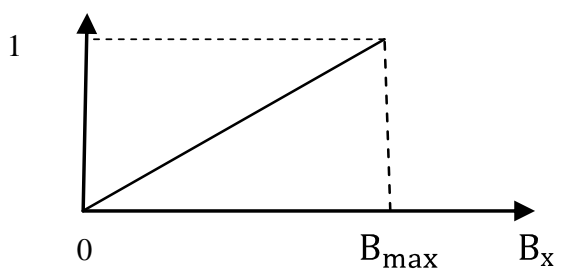

Figure 3. Normalization function of Band width

Normalization function for velocity $\mathrm{N}(\mathrm{v})$ is defined by equation (5).

$\mathrm{N}_{\mathrm{V}}=\left\{\begin{array}{c}0, \mathrm{~V}_{\mathrm{x}}>\mathrm{V}_{\text {max }} \\ 1-\frac{\mathrm{V}_{\mathrm{x}}}{\mathrm{V}_{\max }}, 0 \leq \mathrm{V}_{\mathrm{x}} \leq \mathrm{V}_{\max }\end{array}\right.$

Where $V_{X}$ is he velocity with which the mobile is moving and $\mathrm{V}_{\text {max }}$ is the maximum velocity supported by the candidate base station. The normalization function of velocity is plotted in Figure 4. 


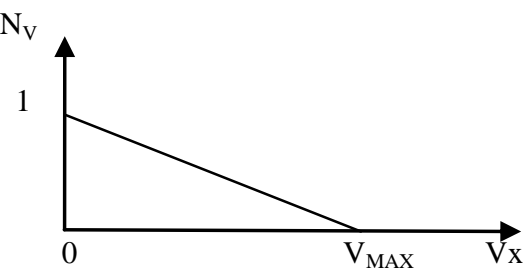

Figure 4. Normalization function of velocity

Similarly normalization function of user preference is defined by equation (6).

$$
N_{p}=\left\{\begin{array}{c}
0, \quad P_{x}>P_{\max } \\
\frac{P_{x}}{P_{\max }}, 0 \leq P_{x} \leq P_{\max }
\end{array}\right.
$$

Where $P_{X}$ is the preference selected by the user and $P_{\text {max }}$ is the maximum preference that can be given to a base station.The normalization function of User Preference is plotted in Figure 5.

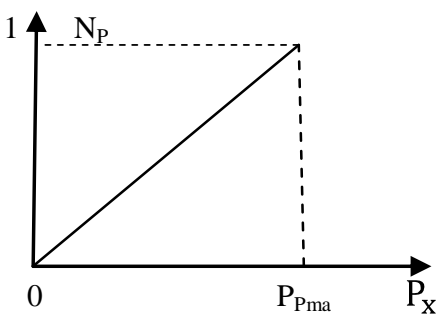

Figure 5. Normalization function of User Preference

\section{COMPUTATION OF THE WEIGHT VECTOR}

The weight of an attribute ' $i$ ' denoted by $W^{i}$ indicates the importance of the attribute. The weight vector is represented as $\left[\mathrm{w}^{\mathrm{RSS}}, \mathrm{w}^{\mathrm{C}}, \mathrm{w}^{\mathrm{BW}}, \mathrm{w}^{\mathrm{V}}, \mathrm{w}^{\mathrm{P}}\right]$ and $W^{i}$ is given by equation (7).

$W^{i}=\frac{\sigma_{i}}{\sum_{i=1}^{5} \sigma_{i}}$

Where $\sigma_{i}$ is the standard deviation of the normalization function values of all the candidate base stations for the given parameter ' $i$ '. Table 1 shows the weight vector corresponding to the given set of normalization function values.

\section{EVALUATION OF NETWORK SELECTION FUNCTION}

Now the Network Selection function (NSF) for each candidate network is evaluated using equation (8).

$f=\sum_{i=1}^{5} N_{i} * w_{i}$

After evaluating the NSF for all the candidate networks, the decision is made as follows:

The $\mathrm{k}^{\text {th }}$ network is selected if

$N S F_{k}=\max \left\{N S F_{1}, N S F_{2}, \ldots . N S F_{n}\right\}$
The Network having highest NSF value is considered as the target network.

\section{SIMULATION MODEL AND RESULTS}

Our simulation scenario first assumes that the coverage area of Wi-Fi is overlapped by Wi-Max which in turn overlapped by CDMA network as shown in Figure 6. The mobile node which is multi-mode capable, initially belongs to CDMA network and starts moving towards Wi-Max and then towards Wi-Fi and stops there. We assume that the input parameters required for network selection algorithm are acquired from the network and mobile terminal.

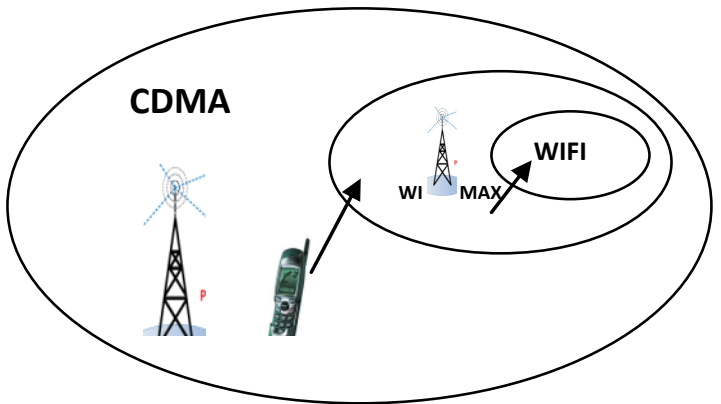

Figure 6. Movement Pattern of Mobile Node

When the mobile node moves from CDMA network towards WiMax network, the network selection module in the mobile terminal acquires the input parameters and normalized values of these parameters are determined. Then network selection function value is computed for the available networks. Due to complexity reasons, we have directly considered the normalized values of the parameters.

First consider the mobile which is originally associated with CDMA network has moved towards the Wi-Max network. The NSF computation for all the networks is as shown in Table 1.

Table 1. NSF when the mobile is under the coverage of Wi-Max

\begin{tabular}{|c|c|c|c|c|c|c|}
\hline & RSS & C & BW & V & P & NSFValue \\
\hline Wi-Fi & 0 & 0.8 & 0.6 & 0.2 & 0.6 & 0.373 \\
\hline $\begin{array}{c}\text { Wi- } \\
\text { Max }\end{array}$ & 0.8 & 0.6 & 0.6 & 0.5 & 0.5 & 0.639 \\
\hline CDMA & 07 & 0.2 & 0.2 & 0.7 & 0.4 & 0.475 \\
\hline Weight & 0.33 & 0.231 & 0.174 & 0.19 & 0.755 & ------ \\
\hline
\end{tabular}

Next consider the case where the mobile terminal is moving towards the Wi-Fi network and is under the coverage of both WiFi, Wi-Max networks. NSF for all the three networks is as shown in Table 2. As the Wi-Fi network is having the maximum NSF value, it is selected as the target network. The graph plotted 
between Movement of the mobile node and NSF value of each network is shown in Figure 7.

\section{CONCLUSIONS AND FUTURE WORK}

In this paper we presented a network selection algorithm based on Fuzzy Multiple Criteria Decision making that calculates the quantitative value of each normalized parameter and finds the weight of each quantitative value. This weight vector together with normalized parameter values are used to evaluate Network Selection Function of the available networks. The network with highest NSF is selected as the target network.

Since the algorithm is taking mobility parameters like speed of the mobile and User preference into account, it supports seamless mobility and reduces unnecessary handoffs. Further the performance of the algorithm can be improved by taking considering a threshold on the NSF value in order to prevent frequent handoffs.

Table 2. NSF when the mobile is under the coverage of Wi-Fi

\begin{tabular}{|l|l|l|l|l|l|l|}
\hline & RSS & C & BW & V & P & NSF \\
\hline Wi-Fi & 0.8 & 0.8 & 0.6 & 0.2 & 0.6 & 0.514 \\
\hline Wi-Max & 0.5 & 0.6 & 0.6 & 0.3 & & 0.446 \\
\hline CDMA & 0.6 & 0.2 & 0.2 & 0.7 & 0.4 & 0.384 \\
\hline Weight & 0.15 & 0.199 & 0.219 & 0.251 & 0.095 & ------ \\
\hline
\end{tabular}

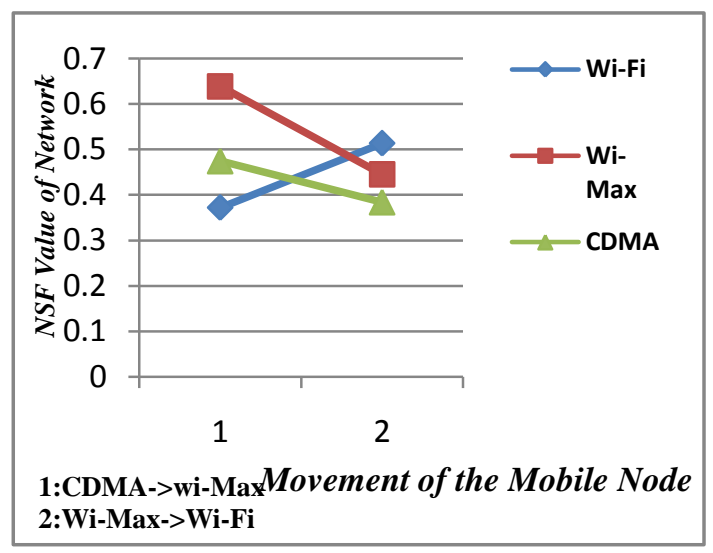

Figure 7. movement of the mobile node Vs NSF Values

\section{REFERENCES}

[1] Suk yu Hui and Kai Hau Yeung, "Challenges in the migration to 4G mobile systems", IEEE Communications Magazine, December 2003, pp.54-59.

[2] Christian Makaya and Samuel pierre, "An Architecture for Seamless Mobility Support in IP-Based Next Generation Wireless Networks", IEEE Transactions on Vehicular Technology, Vol.57, No.2, March 2008, pp.1209-1225.

[3] Duk kyung Kim, David Griffith and Nada Golmie, "A new call Admission control scheme for Heterogeneous wireless networks", IEEE Transactions on Vehicular Communications vol.9, No.10, October 2010,pp. 3000-3005.

[4] Ramon Ferrus, Oriol Sallent and Ramon Agusti "Interworking In Heterogeneous Wireless Networks: Comprehensive Framework And Future Trends", IEEE Wireless Communications, April 2010, pp. 22-31.

[5] Chi Sun, Enrique Stevens-Navarro and Vincent W.S. Wong," A constrained MDP based vertical handoff decision algorithm for $4 \mathrm{G}$ Wireless Networks, pp. 2169-2174, proceedings of ICC 2008.

[6] Hongwei Liao, Ling Tie and Zhao Du, "A Vertical Handoff Decision Algorithm based on Fuzzy Control Theory", Proceedings of the First International Multi-symposiums on Computer and Computational Sciences, 2006.

[7] K.Ayyappan and P.Dananjayan, "RSS Measurement for Vertical Handoff in Heterogeneous network" pp. 989-994, Journal of Theoritical and Applied Information Technology, 2005-2008.

[8] Vivek Gupta, "Mobility Using IEEE 802.21 in a Heterogeneous IEEE 802.16/802.11 Based IMT Advanced 4G network:, IEEE Wireless Communications, April 2008.

[9] Kenichi Taniuchi et.al., "IEEE 802.21: Media Independent Handover: Features, Applicability and Realization”, IEEE Communications Magazine, January 2009.

[10] A. De La Oliva, A. Banchs, I. Soto, T. Melia and A. Vidal, “ An Overview of IEEE 802.21: Media Independent Handover services", IEEE Wireless Communications, vol. 15, pp.96$103,2008$.

[11] E. Stevens-Navarro and V.W.S. Wong, "Comparison between vertical Handoff Decision Algorithms for Heterogeneous Wireless Networks" Proceedings of $63^{\text {rd }}$ Vehicular Technology Conference, 2006, pp.947-951. 